\title{
Automatic Face Recognition for Various Expressions and Facial Details
}

\author{
Guru Kumar Lokku, G. Harinatha Reddy, M. N. Giri Prasad
}

\begin{abstract}
Face Recognition Technique (FRT) was a unique Biometric Technique which tries to spot the people from still images or video frames by using techniques of pattern recognition. Face recognition includes both face identification and face verification (authentication). The FR Design system follows two basic steps i.e. Feature extraction and classification of patterns. Automated FR finds many applications in real time environment ranging from Social Media, surveillance to biometric authentications. Many state-of-the-art face recognition techniques had been implemented, but the Automated Face Recognition (AFR) taken by digital cameras in unconstraint real-world environment continues to be terribly difficult, since it involves vital variations in each acquisition conditions, yet as in facial expressions and in pose variations. Thus, this paper presents the theme of computer based automatic face recognition in lightweight of the most contests therein areas with developed solutions that supports applications of signal, image processing and computing strategies.
\end{abstract}

Index Terms: Face acknowledgement, HOG, Machine learning, PCA, Wild.

\section{INTRODUCTION}

In Face Identification procedure, calculations regularly start via scanning for human eyes. Eyes comprise valley area, and are said to be one of the simplest highlights to identify [1]-[4]. When eyes are identified, the calculation may then start to recognize facial areas including eyebrows, the mouth, nose, nostrils and the iris [5]. When the calculation construes that it has distinguished a facial area, it would then be able to apply extra tests to approve whether it has in-fact, recognized a face or not [13]. Therefore, face identification is needed to identify an individual, based on facial image and this facial image will be compared with all the registered persons depending on one-to-many matching algorithm.

The facial verification is aimed to establish a model of a face identity and then to test a data sample against this identity [7]. It is done with confirming a query face image by either accepting or rejecting query face image based on one-to-one matching algorithm.

Face Recognition or acknowledgment depicts a biometric innovation that goes path past perceiving when a human face is available. It really attempts to build up whose face it is. This technique uses computer applications that captures an individual face, retrieves features and looks at it to registered images available in the data base. While facial

Revised Manuscript Received on July 22, 2019.

Mr. Guru Kumar Lokku, Research Scholar, Dept. of E.C.E., J.N.T.U.C.E.A., A.P., India

Dr. G. Harinatha Reddy, Professor, Dept. of E.C.E., N.B.K.R.I.S.T., A.P., India

Dr. M. N. Giri Prasad, Professor, Dept. of E.C.E., J.N.T.U.C.E.A., A.P., India. acknowledgment isn't $100 \%$ precise, it can precisely decide when there is a solid shot that a man's face matches somebody in the database.

Extracting meaningful data features in AFR against a facial image results to better recognition rate. Suppose, the features are related to different classes and distance among them are larger, then these extracted features are necessary for recognizing the inter class facial image.

In this paper, the plan of face acknowledgment framework utilizing Principal Component Analysis (PCA) feature extraction [8] and investigation of the consequences of these strategies for various pose, illumination and expression challenges are exhibited.

\section{CHALLENGES IN RECOGNIZING FACES}

The investigation and examination of face appearances caught by computerized cameras address an extensive variety of challenges, as enumerated in Sections A, B and C, where all directly affect the face detection and recognition system.

\section{A. Pose disparities}

Head actions that was depicted with self-centered flip/turn positions namely pitch, roll and yaw, or ever-changing viewpoints of the camera [15] could prompt generous variations in face look, additionally shape and create intra-subject varieties of faces as showed in Figure 1, creating machine-driven face acknowledgment through Pose assignment
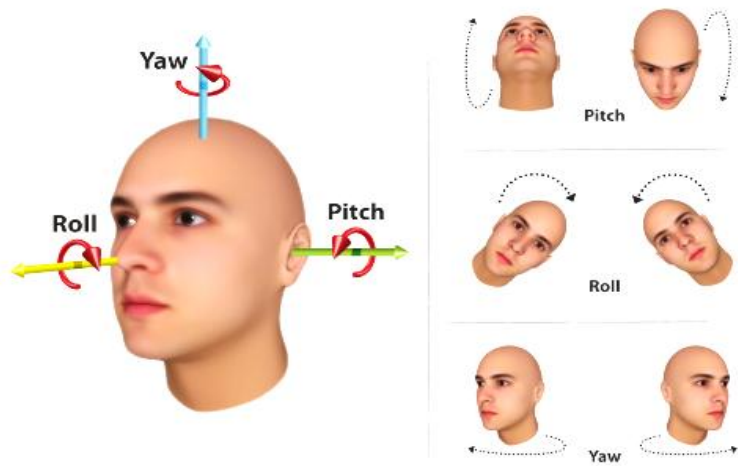

Fig.1. Illustration of head movements around self-centered turn positions, namely (a) pitch, (b) roll and (c) yaw

As AFR was very profound to disparities in pose, correction of pose is necessary and can be accomplished with resources of efficient practices directing to alternate facial coordinates or aligning it to the axis of the images as described [6].
Published By: Blue Eyes Intelligence Engineering \& Sciences Publication 


\section{B. Occlusions / Shaping elements Disparity}

The diversity for within the class facial pictures is due to non-existence of shaping elements (Fig. 2a) or occlusions existence, like beard, moustache (Fig. 2b), cap (Fig. 2c), shades (Fig. 2d), face occlusions like nearer objects, background and so on (Fig. 2e) [14].
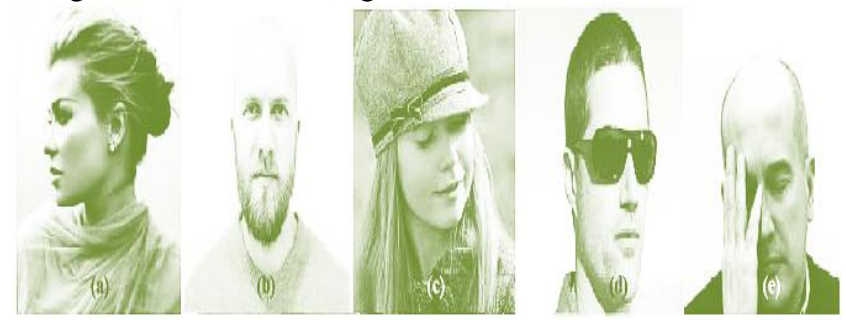

Fig. 2: Existence/Non-Existence of shaping elements/occlusions that reflects (a) absence of shaping elements, (b) moustache $\&$ beard, (c) cap, (d) shades, and (e) face occlusions

Therefore, unrestricted atmosphere oriented facial pictures that were taken normally need efficient face recognition with mask or faces reformed by occlusions or accessories, which can be achieved by means of proper methodologies, for example, texture-based algorithm [12].

\section{Facial expression disparity}

In face appearances, larger inconstancy may occur due to variations of outward appearances instigated by changing individual's passionate situations ${ }^{16}$ which are shown in Fig. 3.

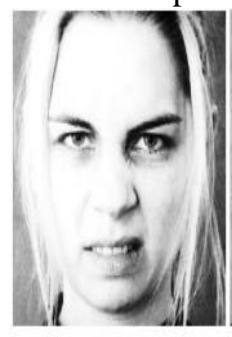

(a)

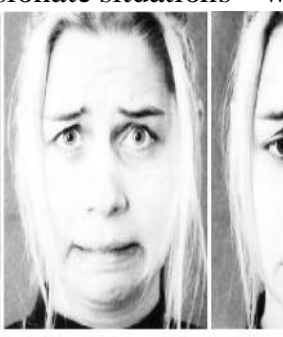

(b)

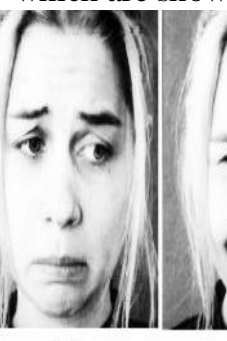

(c)

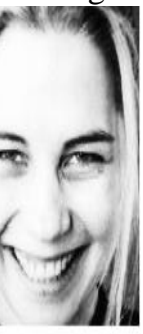

(d)
Fig. 3. facial expressions variations, reflecting (a) anger, (b) disgust, (c) grief, (d) happiness.

Subsequently, by naturally perceiving various outward expressions of face are mutually essential for the assessment of expressive states and the robotized facial acknowledgment. In specific, individual looks results from macro-expressions, that depicts repulsion, satisfaction, irritation, terror, misfortune or astonish, and further automatic, quick facemask examples like micro-expressions; every one of these appearances producing flexible movement of the face.

\section{FACE RECOGNITION SYSTEM}

\section{A. Feature Extraction Technique}

In order to perform AFR, Facial features describe every face in a codified means that are computationally well-organized for additional processes i.e., matching, classification and other techniques namely supervised \& unsupervised learning methods. It is also seen that, extracting facial details from a picture will aid to detect and to trace face inside the image.

\section{B. Principal Component Analysis}

\section{Feature extraction:}

The basic idea is to transform a raw image into a data sample more appropriate for verification

Step 1: Extract the face from the pgm image 9.pgm according to eye centre coordinates.
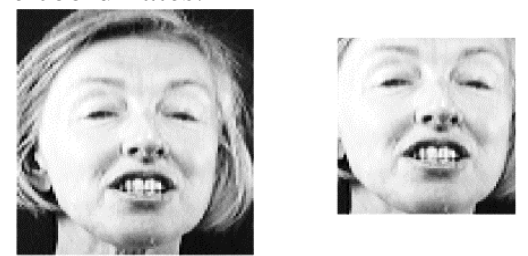

Step 2: Perform geometric normalization, such as scaling and rotation compensation to align the eyes.

Step 3: Perform photometric normalization on the extracted face which scales output pixel values between 0 and 1 , resulting to binary image.

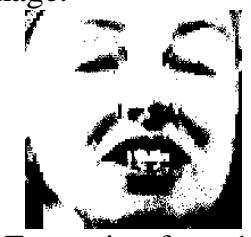

Step 4: PCA Feature Extraction from 112 x 92 Binary Image.

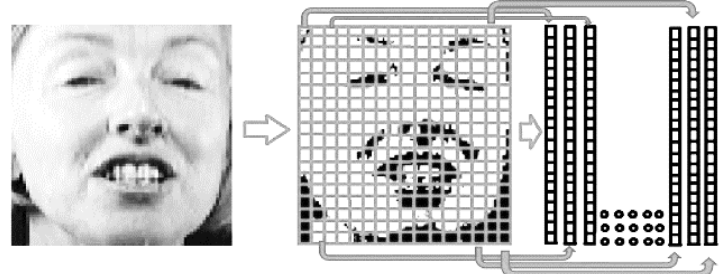

Step 5: Compute the PCA matrix from a list of files.

Step 6: Extract Eigen faces for visualization purposes.

Step 7: Project face images into the Eigen space defined by the Eigen faces that contains $96 \%$ of the variance.

These features extracted from face could range between sparse to dense ones by considering appearance, geometry or texture of the facial image.

\section{Feature matching/ classification}

In this AFR method, characterization is regularly utilized as shown in Figure 4. Certainly, in this machine-learning technique $^{9}$ first features were learnt and then mapping the facial features with the help of functions of a query face to one of the registered images in the database, resulting in a PCA classifier ${ }^{1}$. Classifiers were applied to the whole extracted features of the facial images in order to approximately precise facial characteristics like gender, age, race, etc. One of the most successful AFR methods is the Eigen face Approach, which considers intensity values of picture elements, then after uses $\mathrm{PCA}^{3}$ for the distribution of ghostly faces or Eigen faces by Eigen vectors which characterizes face variations with a set of features contributing to more or less to an Eigen vector. Therefore, each Eigen face ${ }^{2}$ may be treated as an Eigen vector. Also, mapping with the nearest neighbor technique to the probe face projection in the Eigen face space can lead to excellent test-face recognition. 


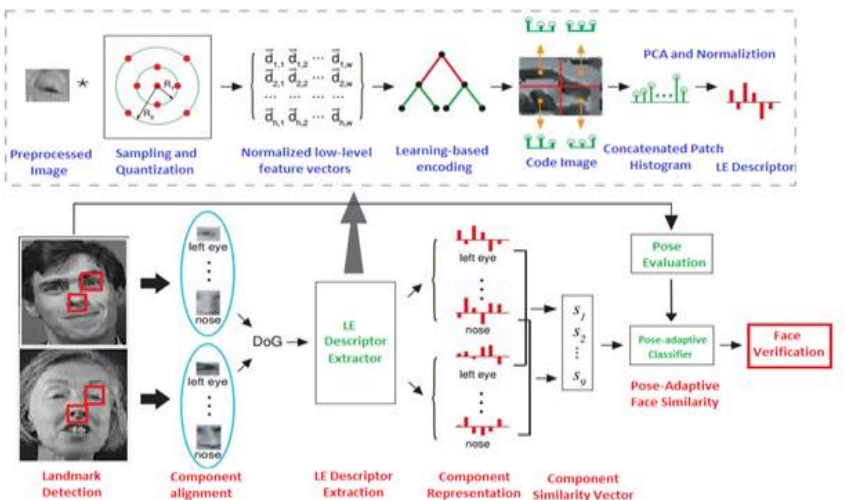

Fig. 4. Schematic Representation Face Detection System

In PCA ' $\mathrm{N}$ ' input variables of same length $\mathrm{K}$ present in the n-dimensional vector $m=\left[m_{l}, \quad m_{2}, \ldots m_{N}\right]^{T}$ was transformed into a vector $n$ given by

$$
n=A\left(m-M_{m}\right)
$$

Here in the vector ' $\mathrm{m}$ ' each row is assumed to be one input, containing ' $\mathrm{L}$ ' values with the mean $\mathrm{M}_{\mathrm{m}}$ represented by

$$
M_{m}=E\{m\}=\frac{1}{L} \sum_{l=1}^{L} m_{l}
$$

Matrix A is the covariance matrix $C_{m}$ that are formed by the Eigen vectors e of $C_{m}$ by

$$
\begin{aligned}
C_{m} & =E\left\{\left(m-M_{m}\right)\left(m-M_{m}\right)^{T}\right\} \\
& =\frac{1}{L} \sum_{l}^{L} m_{l} m_{l}^{T}-M_{m} M_{m}^{T}
\end{aligned}
$$

Since vector ' $\mathrm{m}$ ' is N-dimensional, therefore $C_{m}$ has a dimension of $\mathrm{N} x \mathrm{~N}$. The main diagonal values of $C_{m}(x, y)$ represents variances of $m$ and remaining values are the covariance among input variables $m_{x}, m_{y}$.

$$
\begin{gathered}
c_{m}(x, y)=\left.E\left\{\left(m_{x}-M_{x}\right)^{2}\right\}\right|_{x=y} \\
c_{m}(x, y)=E\left\{\left(m_{x}-M_{x}\right)\left(m_{y}-M_{y}\right)\right\}
\end{gathered}
$$

Also based on Eq. (1) inversion of PCA exists because rows of matrix $A$ are orthonormal.

$$
m=A^{T} n+M_{m}
$$

Apart from matrix theory, PCA kernel finds extensive applications in the field of signal and image processing to computer vision processing.

\section{EXPERIMENT}

Initially, experimental settings of the ORL database were made and then continued to implement various strategies on the challenging issues of facial database.

The AT\&T (formerly "The ORL Database of Faces") Face Database contains 400 grayscale images of forty different individuals in Portable Grey Map (.pgm) format which is designed to be easily exchanged between platforms. There are 10 different images per individual or subject considered with varying face configurations like happy, normal, sad, drowsy, surprised, wink, right light, left light, wearing glasses, and wearing no glasses. Each image has a pixel resolution of $92 \times 112$ considering 256 grey levels per pixel. The challenging facial images are distributed into 40

\begin{tabular}{|c|c|c|c|c|c|c|c|c|}
\hline \multirow{4}{*}{ 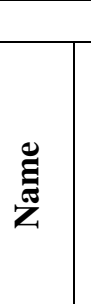 } & \multicolumn{8}{|c|}{ Table 1: Summary of AT\&T Facial Database } \\
\hline & \multirow{3}{*}{$\begin{array}{l}\frac{n}{e} \\
\frac{e}{0} \\
\overline{0}\end{array}$} & \multirow{3}{*}{ 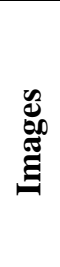 } & \multicolumn{6}{|c|}{ Challenges } \\
\hline & & & \multicolumn{3}{|c|}{ Head Pose } & \multirow{2}{*}{ 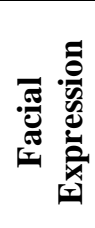 } & \multirow{2}{*}{ 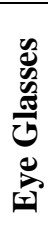 } & \multirow{2}{*}{ 氞 } \\
\hline & & & $\underset{\mathbb{N}}{\mathbb{N}}$ & $\stackrel{乛}{2}$ & $\stackrel{z}{z}$ & & & \\
\hline 응 의 & \& & $\underset{+}{8}$ & $\begin{array}{l}\stackrel{\text { }}{ } \\
+1\end{array}$ & $\begin{array}{l}\text { તิ } \\
+1\end{array}$ & 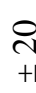 & $\stackrel{0}{2}$ & $\stackrel{\infty}{2}$ & $\stackrel{\infty}{2}$ \\
\hline
\end{tabular}
directories allotting each directory to an individual or subject, which have names of the frame s\#, where \# demonstrates the subject number (running 1 to 40) and in every one of these directories contain ten distinct pictures of that subject, which have names of the shape h.pgm, where $h$ is the picture number for that subject (1 and 10).
Each photo is taken against a dim consistent background with the subjects in an upright, frontal position (with tolerance for some side movement). The subjects are in various positions, namely frontal, up-left/ right along with variations in face emotions, poses and facial details by taking $\pm 20 \%$ pitch, roll, yaw into consideration.

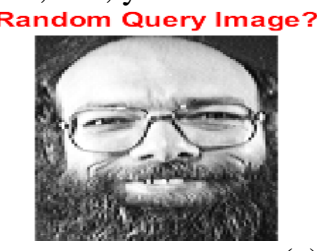

(a)
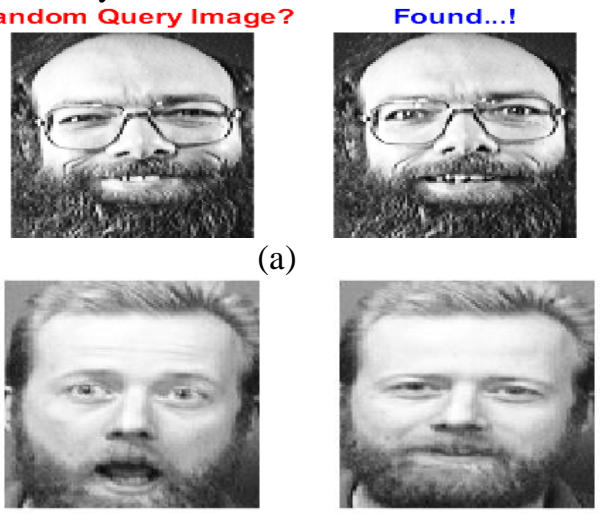

(b)
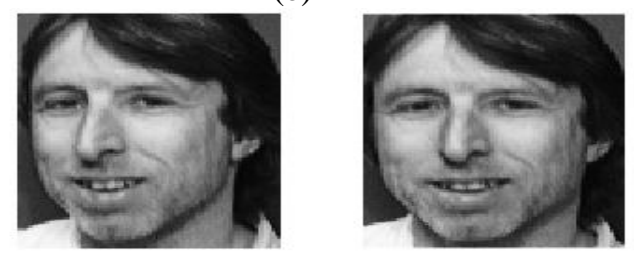

(c)
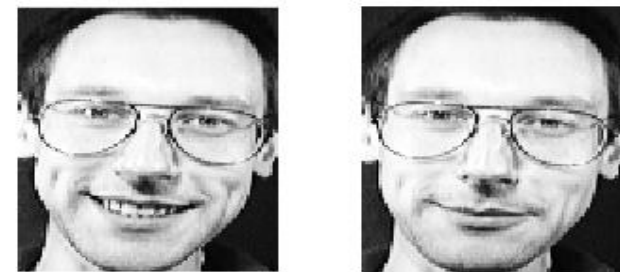

(d)
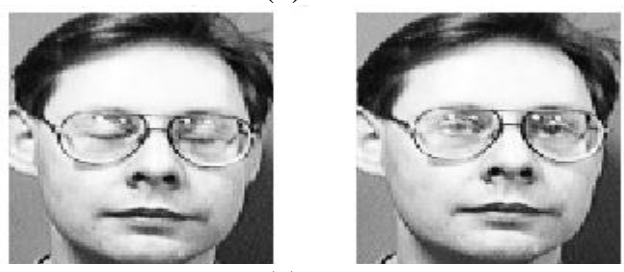

(e) 

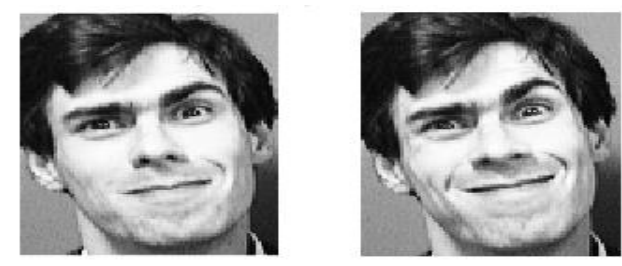

(f)
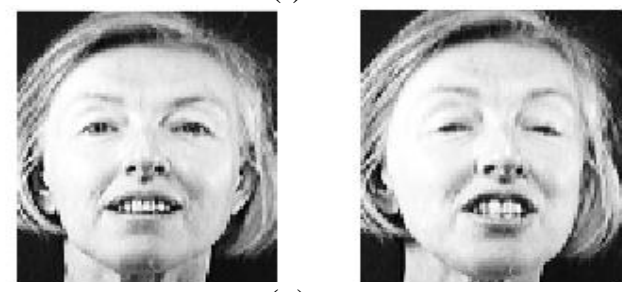

(g)

Fig. 5: Output results found in search of a Random Query Image

\section{A. Analysis:}

Fig. 5.a.: The random query image in the above figure contains shades which may be treated as an occlusion. From the founded image we can conclude that query image was absolutely matched even with variation in expression.

Fig. 5.b.: This figure depicts that the random query image is emotion oriented which is tougher task for recognition but still this method is able to achieve better recognition rate.

Fig. 5.c.: This figure portrays a side faced random query image with less than $20 \%$ yaw and face was recognized successfully.

Fig. 5.d.: This figure represents query image with 5\% roll angle with smiling emotion as input and the individual is identified from the database.

Fig. 5.e.: This figure shows occlusion-based query image with shades and slightly closed eyes and face recognition is achieved successfully.

Fig. 5.f.: The above query face image was tilted towards upside right with happiness as facial emotion and face was detected with correct identity.

Fig. 5.g.: The query image mentioned above was a normal frontal face image and was matched with other image in the database possessing various expression with slightly eyes closed.

Based on the methodology implemented, an image is taken as a query image at random among the available 400 images with 40 subjects of 10 different poses, illumination, expression and this query image features have been extracted using PCA, where the Eigen faces are treated as a column Eigen vector. Each Eigen vector column is treated as a single image. The processing time of query image takes $2-6 \mu$ sec and also this query image is compared against the remaining images present in the data base. If a cluster of features of an image present in database are identified similar to the query image features, then verification is done by matching the two images. Approximately the total recognition rate is identified as $90 \%$ even with $\pm 20 \%$ in pitch, roll and yaw.

\section{CONCLUSION}

This paper, aimed to propose Automated Face Recognition for various expressions and facial details in order to overcome recognizing face under constraint environment having various pose, constant illumination and various expression. The computed results say that the proposed AFR was superior to the state-of-art methods. In nearby future, the same framework can be applied to even wild scenario with unconstrained environment.

\section{REFERENCES}

1. M. A. Abuzneid and A. Mahmood, "Enhanced Human Face Recognition Using LBPH Descriptor, Multi-KNN, and Back-Propagation Neural Network," in IEEE Access, vol. 6, pp. 20641-20651, 2018

2. C. Ding and D. Tao, "Trunk-Branch Ensemble Convolutional Neural Networks for Video-Based Face Recognition," in IEEE Transactions on Pattern Analysis and Machine Intelligence, vol. 40, no. 4, pp. 1002-1014, 1 April 2018.

3. J. Lei, Q. Han, L. Chen, Z. Lai, L. Zeng and X. Liu, "A Novel Side Face Contour Extraction Algorithm for Driving Fatigue Statue Recognition," in IEEE Access, vol. 5, pp. 5723-5730, 2017.

4. S. P. Mudunuri and S. Biswas, "Low Resolution Face Recognition Across Variations in Pose and Illumination," in IEEE Transactions on Pattern Analysis and Machine Intelligence, vol. 38, no. 5, pp. 1034-1040, 1 May 2016.

5. A.Punnappurath, A. N. Rajagopalan, S. Taheri, R. Chellappa and G Seetharaman, "Face Recognition Across Non-Uniform Motion Blur, Illumination, and Pose," in IEEE Transactions on Image Processing, vol. 24, no. 7, pp. 2067-2082, July 2015.

6. F. Juefei-Xu, K. Luu and M. Savvides, "Spartans: Single-Sample Periocular-Based Alignment-Robust Recognition Technique Applied to Non-Frontal Scenarios," in IEEE Transactions on Image Processing, vol. 24, no. 12, pp. 4780-4795, Dec. 2015.

7. W. Deng, J. Hu, J. Lu and J. Guo, "Transform-Invariant PCA: A Unified Approach to Fully Automatic Face Alignment, Representation, and Recognition," in IEEE Transactions on Pattern Analysis and Machine Intelligence, vol. 36, no. 6, pp. 1275-1284, June 2014.

8. H. T. Ho and R. Chellappa, "Pose-Invariant Face Recognition Using Markov Random Fields," in IEEE Transactions on Image Processing, vol. 22, no. 4, pp. 1573-1584, April 2013

9. M. Yang, L. Zhang, S. C. Shiu and D. Zhang, "Robust Kernel Representation With Statistical Local Features for Face Recognition," in IEEE Transactions on Neural Networks and Learning Systems, vol. 24, no. 6, pp. 900-912, June 2013.

10. W. W. W. Zou and P. C. Yuen, "Very Low-Resolution Face Recognition Problem," in IEEE Transactions on Image Processing, vol. 21, no. 1, pp. 327-340, Jan. 2012.

11. Geng, Z. Zhou and K. Smith-Miles, "Individual Stable Space: An Approach to Face Recognition Under Uncontrolled Conditions," in IEEE Transactions on Neural Networks, vol. 19, no. 8, pp 1354-1368, Aug. 2008

12. X. Chai, S. Shan, X. Chen and W. Gao, "Locally Linear Regression for Pose-Invariant Face Recognition," in IEEE Transactions on Image Processing, vol. 16, no. 7, pp. 1716-1725, July 2007.

13. M. Pantic and I. Patras, "Dynamics of facial expression: recognition of facial actions and their temporal segments from face profile image sequences," in IEEE Transactions on Systems, Man, and Cybernetics, Part B (Cybernetics), vol. 36, no. 2, pp. 433-449, April 2006.

14. Dagher and R. Nachar, "Face recognition using IPCA-ICA algorithm," in IEEE Transactions on Pattern Analysis and Machine Intelligence, vol. 28 , no. 6, pp. 996-1000, June 2006

15. M.Turk, A.P. Pentland, "Face Recognition Using Eigenfaces", Proc. IEEE Conf. Computer Vision and Pattern Recognition, pp. 586-591, 1991.

16. Y. Chen, C. Han, C. Wang and K. Fan, "Face Recognition Using Nearest Feature Space Embedding," in IEEE Transactions on Pattern Analysis and Machine Intelligence, vol. 33, no. 6, pp. 1073-1086, June 2011 . 


\section{AUTHORS PROFILE}

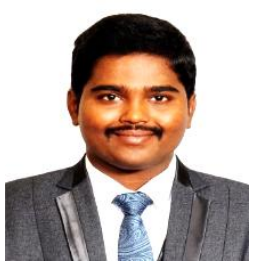

Mr. Guru Kumar Lokku received his B.Tech. degree in Elect. \& Comm. Engg. from S.V. University in 2009, M.E. degree in VLSI Design from Anna University in 2012. He is currently a full time Research Scholar in JNT University Anantapur. His areas of interests include Computer Vision \& Digital Image Processing, VLSI Design, Embedded System and IOT. He is a member of professional societies like ISTE, ISRD and IAENG.

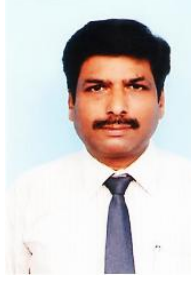

Dr. G. Harinatha Reddy received his Ph.D. degree from Jawaharlal Nehru Technological University, Hyderabad, India, in 2010. Currently, he is a Head \& Professor in the ECE Department, N.B.K.R. Institute of Science \& Technology, Vidyanagar, India. His areas of interests include Signal Processing, Biomedical Instrumentation, Biomedical Signal and Image Processing. Also he is a member in professional societies like the ISTE, IE and IETE.

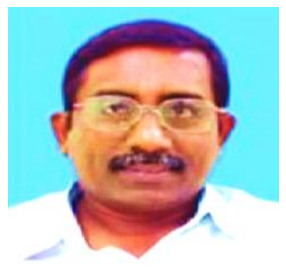

Dr. M. N. Giri Prasad received the Ph.D. degree from Jawaharlal Nehru Technological University, Hyderabad, India, in 2003. He is a Professor with ECE Department, Jawaharlal Nehru Technological University Anantapur, Ananthapuramu, India. His areas of interests are Wireless communication, Biomedical Instrumentation, Digital Image Processing, VHDL Coding, Evolutionary Computing, Biomedical Signal, and Image Processing. He is currently a Director of Admissions for Jawaharlal Nehru Technological University Anantapur, also he is a member in professional societies like the ISTE, the IEI, and the NAFEN. 